\title{
Comparison of Dilapan-S and laminaria for cervical priming before surgical pregnancy termination at 17-22 weeks' gestation
}

This article was published in the following Dove Press journal:

International Journal of Women's Health

19 October 2011

Number of times this article has been viewed

\section{Dennis G Chambers \\ Robin J Willcourt \\ Anthony R Laver \\ Jane K Baird \\ Wye Y Herbert}

The Queen Elizabeth Hospital

Pregnancy Advisory Centre, Woodville

Park, Adelaide, South Australia, Australia
Correspondence: Dennis G Chambers Pregnancy Advisory Centre, 2I Belmore Terrace, Woodville Park, Adelaide, South Australia 5064, Australia

Tel +6I 882433999

Fax +6I 882433998

Email dennis.chambers@health.sa.gov.au
Methods: A retrospective analysis of medical records of three consecutive cohorts of women. All cohorts received a digoxin feticide injection on Day 1 . Two cohorts were treated with laminaria, cohort A of 151 women over 1-2 days and cohort B of 52 women over 1-3 days, and cohort $\mathrm{C}$ of 151 women was treated with Dilapan-S over 1-3 days.

Results: Adequate cervical priming for dilatation and evacuation (D\&E) on Day 2 was achieved in $98 \%$ of the Dilapan-S cohort and $56 \%$ of cohort A and $40 \%$ of the cohort B laminaria cohorts. Return to theater for D\&E 3-4 hours after dilator insertion on Day 2 occurred in $62.3 \%$ of Dilapan-S cohort $\mathrm{C}$ and $9.3 \%$ of cohort A and $4 \%$ of cohort B laminaria cohorts $(P=0.001)$. A mean D\&E theater time of 19 minutes for laminaria cohort A was reduced by $10.1 \%$ in the Dilapan-S cohort $\mathrm{C}(P=0.02)$. The incidence of unscheduled overnight delivery outside the clinic was $0 \%$ for Dilapan-S and 1.3\% for cohort A and 3.8\% for cohort B laminaria cohorts $(P=0.14)$.

Conclusion: Dilapan-S osmotic dilators are superior to laminaria in producing more cervical priming and dilatation in a shorter time. This enables 17-22 week D\&E procedures to be carried out in fewer days and in shorter theater times. They also eliminate the risk of an unscheduled overnight delivery outside the clinic.

Keywords: late second-trimester surgical abortion, dilatation and evacuation, cervical priming, osmotic dilators, Dilapan-S, laminaria tents

\section{Introduction}

The Pregnancy Advisory Centre is a government-funded day surgery clinic providing over 2500 surgical and medical terminations of pregnancy each year in South Australia, Australia. Second-trimester surgical termination by dilatation and evacuation (D\&E) of pregnancies up to 22 weeks' gestation has been performed in this clinic using misoprostol and osmotic cervical dilators for cervical priming since 1994. Misoprostol alone has been used at 13-16 weeks' gestation, and misoprostol as an adjunct to osmotic dilators at 17-22 weeks' gestation. The incidence of these terminations has remained fairly constant over this period with approximately $9 \%$ of all terminations occurring at 13-16 weeks' gestation and 4\% at 17-22 weeks' gestation, figures much in line with published figures overseas. ${ }^{1}$ At $13-16$ weeks, women can be safely terminated in a 1-day D\&E procedure with misoprostol alone for cervical priming. ${ }^{2}$ Although many women at 17-18 weeks can be terminated in 1 day, an increasing proportion of later gestations cannot be adequately cervically dilated for a safe D\&E procedure in 1 day. ${ }^{3}$ Therefore, in the Pregnancy Advisory Centre clinic, all 17-22 weeks' gestation women are treated as potentially multiday procedures with a combination of 
misoprostol and osmotic cervical dilators. Over the first 10 years of this clinic, increasing experience allowed the dosage and timing of the misoprostol to be gradually developed to the regimen that is now used.

The routine use of digoxin feticide began in 2004 because a macerated fetus is more easily evacuated at D\&E. ${ }^{4}$ It was determined that the majority of women prefer the reassurance that the fetus will not be alive at D\&E and that should an unscheduled precipitate delivery occur at home the fetus will not be born alive. For the first 5 years the digoxin was administered on Day 1 without anesthesia. In July 2009, as some women found this procedure distressing, anesthesia on Day 1 was introduced as a routine, the digoxin being injected into the fetal body under ultrasound guidance. At the same time, advantage was taken of the opportunity to commence the insertion of one or two laminaria tent osmotic cervical dilators. After 52 cases it became apparent that this new regimen had not resulted in any reduction in the number of days' preparation required before D\&E. In February 2010 the synthetic osmotic dilator Dilapan-S ${ }^{\mathrm{TM}}$ (Fema International Dilapan Distributors: regional offices listed on www.dilapan.com) was substituted for laminaria to test for possible advantages over laminaria.

With the permission of the Therapeutic Goods Administration, the addition of mifepristone $200 \mathrm{mg}$ to misoprostol and laminaria was trialled between the two laminaria cohorts to enhance cervical dilatation, but after 21 cases the mifepristone was ceased due to a $19 \%$ incidence of cervical lacerations and one overnight delivery at home. After the use of Dilapan-S in 151 late second-trimester women we decided to audit our results, and this number decided the size of the major cohorts. We have been unable to find any published trials comparing Dilapan-S with laminaria. Because of this lack of published data comparing Dilapan-S and laminaria for late second-trimester terminations, we decided to research the outcomes in our clinic of comparable procedures using the two types of osmotic cervical dilators, to determine what clinical benefits Dilapan-S might offer.

\section{Materials and methods}

The study design was a retrospective review of the clinical records of three cohorts of consecutive cases of women undergoing surgical termination of pregnancy at 17-22 weeks' gestation. Cohort A was of 151 women who on Day 1 underwent digoxin feticide injection without anesthesia. On Day 2, three doses of two misoprostol $200 \mu \mathrm{g}$ tablets were administered sublingually 30 minutes apart. Three hours after the last dose, under intravenous propofol anesthesia, the cervix was dilated with rigid Hawkin-Ambler dilators to a dilatation equal to the weeks of gestation in millimeters less $2 \mathrm{~mm}$, as in Table 1. This minimum dilatation requirement before the insertion of osmotic dilators has been determined from experience over the 17 years that we have used this regimen in our clinic, and its purpose is to ensure adequate subsequent cervical priming for safe D\&E the same or next day. The dilatation was followed by artificial rupture of the membranes, drainage of the liquor, and the insertion of $8 \mathrm{~mm}$ laminaria tents, the number equalling the weeks of gestation minus 11. If no rigid dilatation was needed, the misoprostol alone having produced the target dilatation, the woman was considered safe for return to theater the same day for D\&E. Therefore, at the end of the procedure, two moistened misoprostol $200 \mu \mathrm{g}$ tablets were inserted rectally, this route ensuring a rapid response. On return to the recovery ward, one misoprostol $200 \mu \mathrm{g}$ tablet was administered sublingually every 30 minutes for four doses. D\&E was then performed 2-3 hours later under intravenous propofol anesthesia after ensuring that the dilatation of the cervix achieved was appropriate for the gestation as detailed in Table 1, again a safe minimum determined from 17 years' experience in our clinic. Women with inadequate cervical priming by the initial misoprostol were precluded from returning for D\&E on Day 2 and returned the next morning. On Day 3, three doses of two misoprostol $200 \mu \mathrm{g}$ tablets were again administered sublingually 30 minutes apart. Three hours after the last dose, D\&E was performed under intravenous propofol anesthesia.

Cohort B was of 52 women who on Day 1 underwent digoxin feticide injection under propofol anesthesia, followed by the insertion into the cervical canal of one or two laminaria tents. On Day 2, misoprostol $200 \mu \mathrm{g}$ tablets were administered, two sublingually for three doses at half-hour intervals. Half an hour to 1 hour after the last dose the woman was taken to theater for D\&E if the cervix was sufficiently dilated as detailed in Table 1, and further laminaria if not. If dilatation was inadequate, the membranes were ruptured and the liquor drained before further laminaria were inserted. Subsequent

Table I Minimum millimeter $(\mathrm{mm})$ cervical dilatation required at each gestation before osmotic dilator insertion on the morning of Day 2 and before dilatation and evacuation (D\&E) procedure

\begin{tabular}{|c|c|c|c|c|c|c|}
\hline Gestation in weeks & 17 & 18 & 19 & 20 & 21 & 22 \\
\hline $\begin{array}{l}\text { Cervical dilatation in } \mathrm{mm} \\
\text { before osmotic dilator } \\
\text { insertion on Day } 2\end{array}$ & 15 & 16 & 17 & 18 & 19 & 20 \\
\hline $\begin{array}{l}\text { Cervical dilatation in } \mathrm{mm} \\
\text { before D\&E operation }\end{array}$ & 18 & 20 & 22 & 24 & 26 & 28 \\
\hline
\end{tabular}


treatment was the same as in the 151 laminaria cohort described previously. After 52 cases, concern was raised that the number of women needing a 3-day procedure had actually risen rather than fallen, and Dilapan-S was then substituted for laminaria to determine whether this would reduce the number of women needing to return on the third day.

In cohort $\mathrm{C}, 151$ women had $4 \mathrm{~mm} \times 65 \mathrm{~mm}$ Dilapan-S instead of laminaria. As in cohort B, Day 1 consisted of a digoxin feticide injection and the insertion of osmotic dilators under propofol anesthesia, one Dilapan-S dilator being inserted at 17-19 weeks' gestation and two dilators at 20-22 weeks' gestation. On Day 2, misoprostol $200 \mu \mathrm{g}$ tablets were administered, two sublingually for three doses at half-hour intervals, and the woman was taken to theater $1 / 2-1$ hour after the last dose for D\&E if the cervix was sufficiently dilated as detailed in Table 1, and further osmotic dilators if not. If further osmotic dilatation was required, after rupturing the membranes and draining the liquor, Dilapan-S dilators were inserted, two or three at 17-19 weeks and four or five at 20-22 weeks, under propofol anesthesia, followed by the same misoprostol routine as in the laminaria cohorts when return to theater for D\&E the same day was planned. Three hours after the last dose of misoprostol, the woman was returned to theater for D\&E. All D\&E procedures at our clinic are performed under intravenous propofol anesthesia with the addition of an intracervical block of lignocaine local anesthetic with adrenaline. Routine intraoperative ultrasound guidance is used, allowing the operator to see instruments in the uterus and direct them to the fetal parts safely.

Medical records were examined and data were collected relating to parity, gestation, the number of procedures, the number of days of treatment, the D\&E theater time for each woman in each cohort, and any complications. The theater times were used rather than operation times, as these were collected with more strict precision, the time of entry into and exit from the operating theater being collected and recorded separately by both the anesthetist and theater nurse with cross-checking. All the procedures analyzed were carried out by operators with many years of experience. The demographic make-up of all three cohorts was similar. The statistical analysis was performed using Fisher's exact test.

\section{Results}

The number of women in each cohort completing a D\&E on the morning of Day 2, with a breakdown by parity, is shown in Table 2. In this table the number of women in each of the laminaria cohorts was reduced by 2 because of two unscheduled out-of-clinic deliveries overnight in each of the laminaria cohorts. There were no unscheduled deliveries outside the clinic for Dilapan-S, and two deliveries for each of the laminaria cohorts, an incidence of $1.3 \%$ for cohort $\mathrm{A}$ and $3.8 \%$ for cohort $\mathrm{B}(P=0.14)$.

The number of women in each cohort completing a D\&E on the morning of Day 2 was 70 of $149(47 \%)$ in laminaria cohort A, 54 of 151 (36\%) in the Dilapan-S cohort C, and 18 of $50(36 \%)$ in laminaria cohort $\mathrm{B}$. The number of women in each cohort completing a D\&E after 2 days was 148 of 151 $(98 \%)$ in Dilapan-S cohort C, 84 of $149(56 \%)$ in laminaria cohort A, and 20 of $50(40 \%)$ in laminaria cohort B. The advantage of the Dilapan-S cohort over the two laminaria cohorts was $43 \%$ and $59 \%$, respectively.

Return to theater for same-day D\&E 3-4 hours after dilator insertion on Day 2 occurred in 94 of 151 (62.3\%) in Dilapan-S cohort C, 14 of 151 (9.3\%) in laminaria cohort A, and two of $52(3.8 \%)$ in laminaria cohort $\mathrm{B}$, a highly significant Dilapan-S advantage $(P=0.001)$.

The mean D\&E theater times for each cohort are also shown in Table 2. The overall mean D\&E theater time for the three cohorts was 17.1 minutes with Dilapan-S cohort C, 19.0 minutes with laminaria cohort $\mathrm{A}$, and 18.0 minutes with laminaria cohort $\mathrm{B}$. The $\mathrm{D} \& \mathrm{E}$ theater time reduction advantage of Dilapan-S was $10.1 \%$ over laminaria cohort A and $5.1 \%$ over laminaria cohort B $(P=0.02)$.

As well as the two home deliveries in each of the laminaria cohorts, other complications were cervical lacerations requiring suture in $3.4 \%$ (two women) of laminaria cohort $\mathrm{A}$, $5.8 \%$ (three women) of cohort B, and 1.3\% (two women) of Dilapan-S cohort C, and blood loss over $500 \mathrm{~mL}$ in $1.9 \%$ (one woman) of laminaria cohort B and no women in the other two cohorts. There were no perforations, infections, retained products, anesthetic problems, or hospital admissions in any of the cohorts.

The trial of mifepristone in 21 women between the laminaria cohorts resulted in one unscheduled delivery outside the clinic, a $4.8 \%$ incidence, and four cervical lacerations, a $19 \%$ incidence.

\section{Discussion}

From 17 weeks' gestation, an increasing number of women cannot be safely terminated by D\&E in 1 day, misoprostol priming alone becoming progressively inadequate in providing a safe degree of cervical softening and dilatation. A previous study by our clinic determined that inadequate cervical priming increased the risk of perforation of the uterus during late-gestation D\&E. ${ }^{3}$ These gestations therefore require the use of osmotic dilators as an adjunct to misoprostol. We have compared the 
Table 2 Proportion of women undergoing dilatation and evacuation (D\&E) on the morning of Day 2 and mean theater time in each of two laminaria tent cohorts and one Dilapan-S cohort by parity

\begin{tabular}{|c|c|c|c|c|c|c|}
\hline \multirow[t]{4}{*}{ Cohort } & \multicolumn{3}{|c|}{ Nulliparous women } & \multicolumn{3}{|l|}{ Parous women } \\
\hline & Cohort A & Cohort B & Cohort C & Cohort A & Cohort B & Cohort C \\
\hline & I5I women & 52 women & I5I women & I5I women & 52 women & I5I women \\
\hline & laminaria & laminaria & Dilapan-S & laminaria & laminaria & Dilapan-S \\
\hline D\&E morning & 30 of $78(38 \%)$ & 10 of $34(29 \%)$ & 27 of $80(34 \%)$ & 40 of 71 (56\%) & 8 of $16(50 \%)$ & 27 of 71 (38\%) \\
\hline \multicolumn{7}{|l|}{ Day 2} \\
\hline $\begin{array}{l}\text { Mean D\&E } \\
\text { theater time }\end{array}$ & 19.2 mins $(n=78)$ & 17.6 mins $(n=34)$ & 16.6 mins $(n=80)$ & 19.0 mins $(n=71)$ & $17.4 \operatorname{mins}(n=16)$ & 17.0 mins $(n=7 \mid)$ \\
\hline
\end{tabular}

outcomes in three cohorts of women of two laminaria regimens and one Dilapan-S regimen of osmotic dilators. In all cohorts on Day 1 an intrafetal injection of digoxin $1.0 \mathrm{mg}$ was performed to produce fetal demise and maceration. ${ }^{4,5}$ The demographics of the women were similar in all cohorts.

A Cochrane review in 2010 concluded that cervical preparation with osmotic dilators and misoprostol before second-trimester D\&E is safe and effective. ${ }^{6}$ Dilapan-S (Figure 1) is a hydrophilic polymer rod manufactured from Aquacryl, a proprietary hydrogel, which is hygroscopic and expands radially more rapidly, consistently, and to a greater degree than laminaria tents, which are a natural product manufactured from dried seaweed stems (Figure 2). Therefore, fewer Dilapan-S can be inserted for shorter periods of time. Most dilatation occurs within 4-6 hours, and one $4 \mathrm{~mm}$ Dilapan-S produces $7.8-10 \mathrm{~mm}$ dilatation at 2 hours and $12.7-14.6 \mathrm{~mm}$ after 24 hours. The radial pressure on the cervix of the expanding Dilapan-S also provides cervical ripening via prostaglandin synthesis. ${ }^{2}$

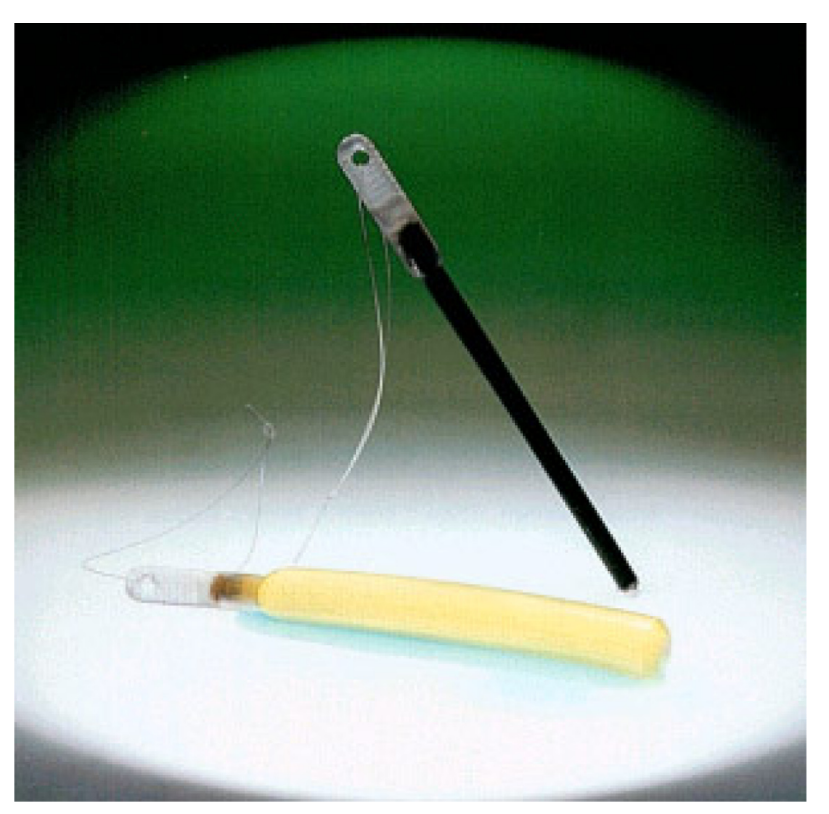

Figure I Dilapan-S before and after hydration.
The main finding of our retrospective study was that the aforementioned characteristics of Dilapan-S enabled us to markedly reduce the number of women who needed to return on Day 3 for D\&E. With Dilapan-S, 98\% of women underwent D\&E on Day 2 as opposed to $56 \%$ in laminaria cohort A and $40 \%$ in laminaria cohort B, the laminaria cohort with the same regimen as the Dilapan-S cohort, an improvement of 1.75-fold and 2.45-fold, respectively.

The number of women ready for D\&E on the morning of Day 2 was $47 \%$ for laminaria cohort A and 36\% each for laminaria cohort B and the Dilapan-S cohort C. The percentage was higher for laminaria cohort A because these women went to theater 3 hours after the last dose of misoprostol, as opposed to the interval being $1 / 2-1$ hour in the other two cohorts. The shorter interval was necessary to allow time for the sameday return to theater for D\&E for women with inadequate cervical priming in the morning. Return to theater for D\&E 3-4 hours after dilator insertion on Day 2 occurred in $62.3 \%$ of Dilapan-S cohort C, 9.3\% of laminaria cohort A, and 3.8\% of laminaria cohort $\mathrm{B}$, a highly significant Dilapan-S advantage $(P<0.001)$. The superiority of the Dilapan-S was due to its greater and more rapid priming of the cervix, enabling return to theater in the early afternoon of the same day for D\&E after insertion of the osmotic dilators in the morning.

Another significant advantage of Dilapan-S was that it eliminated the risk of unscheduled overnight delivery at home, there being none with Dilapan-S and two cases in each of the laminaria cohorts, an incidence of $0 \%$ in the Dilapan-S cohort, $3.8 \%$ in laminaria cohort $\mathrm{A}$, and $1.3 \%$ in laminaria cohort B. As our day surgery clinic is staffed only in office hours, it is unable to treat a woman with an unscheduled delivery out of hours. Such a delivery can be very distressing for a woman and is to be avoided if possible.

A mean D\&E theater time of 19 minutes for laminaria cohort A was reduced by $10.1 \%$ in Dilapan-S cohort $\mathrm{C}$ and by $5.1 \%$ in laminaria cohort $\mathrm{B}$, the Dilapan-S appreciably reducing the theater times due to greater cervical priming $(P=0.02)$. Cervical lacerations needing suture were $1.3 \%$ 


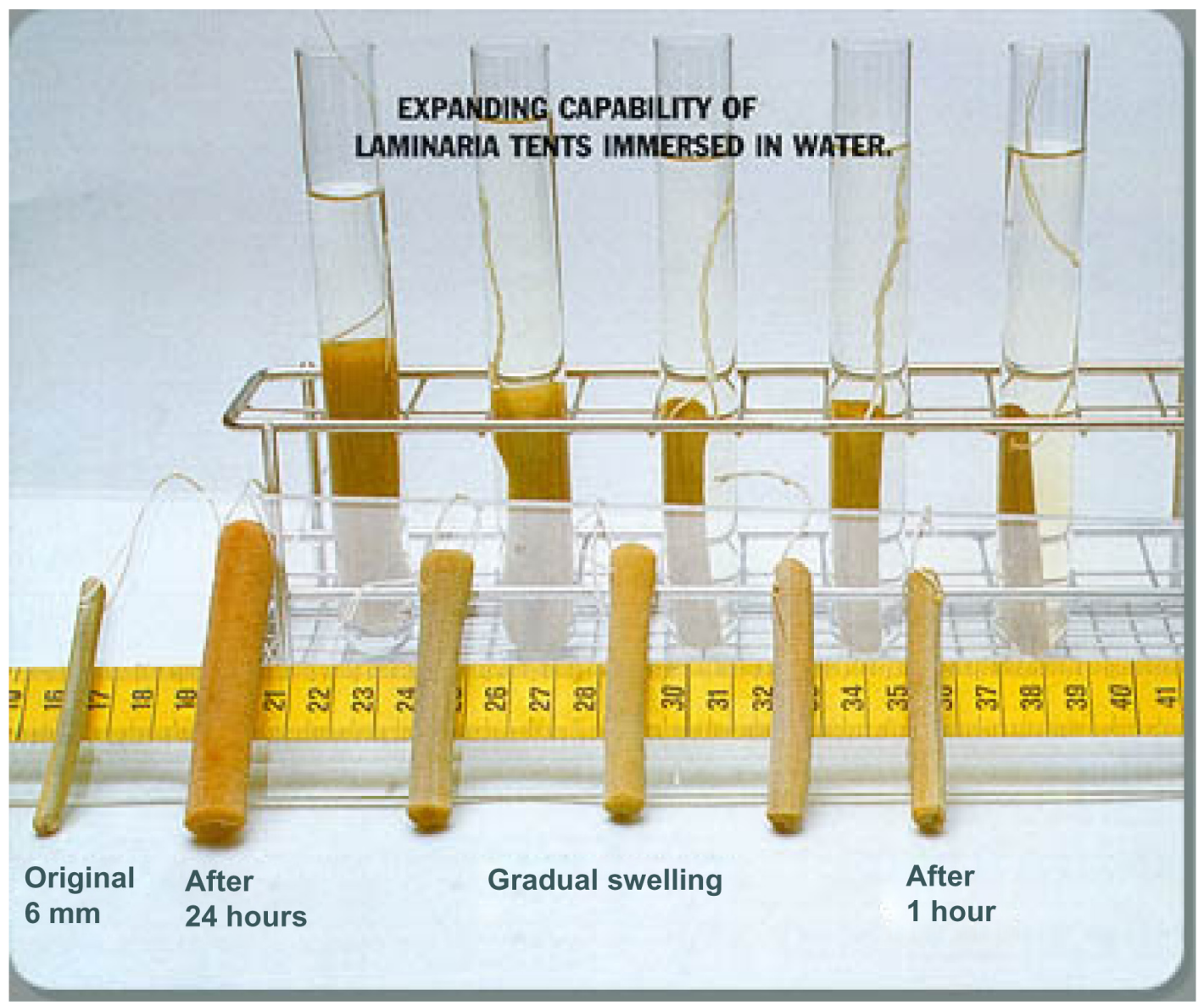

Figure 2 Laminaria tents before and after hydration.

with Dilapan-S cohort C, 3.4\% in laminaria cohort A, and $6 \%$ in laminaria cohort $\mathrm{B}$, again demonstrating the benefit of the greater cervical priming produced by Dilapan-S. There were no serious complications in any cohort. These rates compare favorably with Chasen et $\mathrm{al}^{7}{ }^{7}$ who reported a late second-trimester D\&E complication rate of 5\%. No uterine perforations occurred in the cohorts we studied, which we attribute to our serial use of osmotic dilators. Uterine perforation is a serious complication of second-trimester surgical abortion with a reported incidence of $0.2 \%-0.4 \% .^{2,3}$

Hayes and Fox ${ }^{8}$ have reported that Dilapan-S can be difficult to remove due to entrapment and fragmentation. This problem was not met in the Dilapan-S cohort we studied, which we attribute to our use of the dilators being in graded serial stages over 2 days along with the adjuvant use of high-dosage misoprostol. The misoprostol dosage we have found to be optimally effective is approximately twice that recommended in most published studies. Despite this higher dosage and $20 \%-25 \%$ of our parous women having a cesarean scar, we have never seen a uterine rupture in 17 years of this usage. We attribute this to never exceeding $400 \mu \mathrm{g}$ per dose of misoprostol, with a minimum interval of 30 minutes between doses, and avoiding the vaginal route of administration. Wilson et $\mathrm{al}^{9}$ have compared Lamicel osmotic dilators with misoprostol plus Dilapan-S and shown that although both regimens allowed completion in 1 day for up to 18 weeks' gestation, the former had two cases of hemorrhage requiring hospitalization compared with no serious complications in the misoprostol plus Dilapan-S group.

Our brief trial of mifepristone in 21 women led to one unscheduled out-of-clinic delivery, a $4.8 \%$ incidence, and excessive softening of the cervix with four cervical lacerations, a $19 \%$ incidence of cervical injury. Carbonell et a ${ }^{10}$ have reported that administering mifepristone as an adjunct to misoprostol used for cervical priming before D\&E in 12-20 week second-trimester abortions makes surgery easier and shorter but with the drawbacks of increased cost, more patient clinic visits, and the expulsion of the fetus before $\mathrm{D} \& \mathrm{E}$ being a risk with mifepristone. They pointed out that this has consequences for the woman's health, as this expulsion may occur at any time and place. The Cochrane review has also concluded that mifepristone is associated with high rates of preprocedural 
expulsions and does not appear to be a useful method of cervical preparation before second-trimester D\&E. ${ }^{6}$

Surgical termination of a second-trimester pregnancy has fewer complications, is completed in a shorter time, makes less demand on clinic staff time, and has a lower cost than medical termination. ${ }^{11}$ A significant proportion of women with fetal anomalies or fetal death are treated in hospital by labor induction. Bryant et $\mathrm{al}^{12}$ state that women facing this situation should be offered a choice of methods, as D\&E is significantly safer and more effective than labor induction for second-trimester abortion for fetal indications. The surgical option can be made even more efficient by determining the optimal method of cervical priming. Our study has shown that a regimen combining misoprostol and Dilapan-S osmotic dilators is a move toward this optimum. Our study shows the benefit of misoprostol, when acting over a few hours, as an adjunct to osmotic dilators. In laminaria cohort $A$, where D\&E was performed 3 hours after the last dose of misoprostol, $56 \%$ of parous women were primed for operation, compared with only $38 \%$ of the Dilapan-S cohort where D\&E was 1/2-1 hour after the last dose of misoprostol.

A limitation of our study is that the large laminaria cohort $A$ did not have exactly the same regimen as Dilapan-S, and laminaria cohort $\mathrm{B}$, which exactly matched the regimen of the Dilapan-S cohort C, was only 52 women in size due to its early abandonment because of its failure to improve on any of the measures of outcome of the preceding laminaria cohort A. Nevertheless, the advantages of Dilapan-S were still great enough to achieve statistical significance. Another limitation of our study is that it was retrospective. The use of theater times rather than operation times was therefore unavoidable because with the study being retrospective, operation times had not been recorded with the same precision that would occur in a prospective study. Fortunately, our clinic has a protocol in place for precision in the recording of theater times.

This study showed that Dilapan-S osmotic dilators are superior to laminaria in producing more cervical priming and dilatation in a shorter time. This enables 17-22 week D\&E procedures to be carried out in fewer days and in shorter theater times. It also eliminates the risk of an unscheduled overnight delivery outside the clinic. The higher cost of Dilapan-S over laminaria tents is offset by the fewer number of dilators required to produce the same dilatation and the shorter duration of treatment required.

\section{Acknowledgment}

We are grateful to Dr John Field, Statistician, Faculty of Health Sciences, University of Adelaide, for statistical analysis.

\section{Disclosure}

This clinical audit study conformed to the standards required for Australian National Health and Medical Research Council exclusion for ethics approval. There is no conflict of interest with any of the authors.

\section{References}

1. Gamble SB, Strauss LT, Parker WY, et al; Centers for Disease Control and Prevention (CDC). Abortion surveillance: United States, 2005. MMWR Surveill Summ. 2008;57:1-32.

2. Lohr PA. Surgical abortion in the second trimester. Reprod Health Matters. 2008;16:151-161.

3. Pridmore BR, Chambers DG. Uterine perforation during surgical abortion: review of diagnosis, management and prevention. Aust $N Z J$ Obstet Gynaecol. 1999;39:349-353.

4. Hern WM. Laminaria, induced fetal demise and misoprostol in late abortion. Int J Gyneol Obstet. 2001;75:279-286.

5. Molaei M, Jones HE, Weiselberg T, et al. Effectiveness and safety of digoxin to induce fetal demise prior to second-trimester abortion. Contraception. 2008;77:223-225.

6. Newmann SJ, Dalve-Endres A, Diedrich JT, et al. Cervical preparation for second trimester dilatation and evacuation. Cochrane Database Syst Rev. 2010;8:CD007310.

7. Chasen ST, Kalish RB, Gupta M, et al. Dilatation and evacuation at $\geq 20$ weeks: comparison of operative techniques. Am J Obstet Gynecol. 2004;190:1180-1183.

8. Hayes JL, Fox MC. Cervical dilatation in second-trimester abortion. Clin Obstet Gynecol. 2009;52:171-178.

9. Wilson LC, Meyn LA, Creinin MD. Cervical preparation for surgical abortion between 12 and 18 weeks gestation using vaginal misoprostol and Dilapan-S. Contraception. 2011;83:511-516.

10. Carbonell JL, Gallego FG, Llorente MP, et al. Vaginal vs sublingual misoprostol with mifepristone for cervical priming in second-trimester abortion by dilatation and evacuation: a randomized clinical trial. Contraception. 2007;75:230-237.

11. Lohr PA, Hayes JL, Gemzell-Danielsson K. Surgical versus medical methods for second trimester induced abortion. Cochrane Database Syst Rev. 2008;23(1):CD006714.

12. Bryant AG, Grimes DA, Garrett JM, Stuart GS. Second-trimester abortion for fetal anomalies or fetal death: labor induction compared with dilatation and evacuation. Obstet Gynecol. 2011;117:788-792.
International Journal of Women's Health

\section{Publish your work in this journal}

The International Journal of Women's Health is an international, peerreviewed open-access journal publishing original research, reports, reviews and commentaries on all aspects of women's healthcare including gynecology, obstetrics, and breast cancer. Subject areas include: Chronic conditions (migraine headaches, arthritis, osteoporosis);

\section{Dovepress}

Endocrine and autoimmune syndromes; Sexual and reproductive health; Psychological and psychosocial conditions. The manuscript management system is completely online and includes a very quick and fair peer-review system. Visit http://www.dovepress.com/ testimonials.php to read real quotes from published authors. 